\title{
EFFECT OF LOW CONCENTRATION OF DHPC ON THE DPPC/SM BILAYER
}

\author{
RODRIGO MONTECINOS ${ }^{l, *}$ STEPHANIE NEIRA-JIMENEZ ${ }^{1}$
}

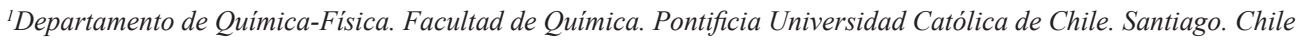

\begin{abstract}
The organization of bilayers depends on the physicochemical and structural properties of the molecules that compose the membrane. The phospholipid molecular shape depends on the relative volumes of their polar headgroups and non-polar acyl chains. In this work, we study the effect of DHPC concentration over the bilayer of large unilamellar vesicles (LUV) prepared with different proportions of dipalmitoylphosphatidylcholine / sphingomyelin / dihexanoylphosphatidylcholine (DPPC/SM/DHPC) using fluorescence spectroscopy and molecular dynamics (MD) simulations. In this work, we use Laurdan's generalized polarization (GP) and 1,6-diphenyl-1,3,5-hexatriene (DPH) fluorescence anisotropy to observe the effect of DHPC concentration on dynamic properties of both, the interface and hydrophobic region. Detailed information, at atomistic scale, was obtained by trajectory calculations of accessible solvent area (ASA), partial densities and order parameters using Molecular Dynamics (MD). The results indicate that DHPC induces global packing defects in the membrane, favoring the liquid crystalline state for the system with higher concentration of DHPC.
\end{abstract}

\section{INTRODUCTION}

Biological membranes are mainly a lipid bilayer in the liquid-crystalline phase. The organization of the bilayer depends on the physicochemical and structural properties of the molecules that compose the membrane. The phospholipid molecular shape depends on the relative volumes of their polar headgroup and apolar acyl chains. Depending on their relative volumes, phospholipids can adopt molecular shapes such as cylindrical, conical or inverted cone. Packing of phospholipids with different molecular shapes can generate different supramolecular organizations, such as micellar, lamellar, hexagonal and cubic phases $^{1,2}$. Different molecular packing of mixed lipids, with different shapes, induces heterogeneous lateral organization, forming microdomains called lipid rafts $^{1,3}$. Moreover, lipid rafts induce spontaneous curvature in the membran $e^{4,5}$. Short-chain phospholipids, such as dihexanoylphosphatidylcholine (DHPC), present an inverted cone shape. This feature allows to generate structures with high mobility and curvature, such as micelles. In the last two decades, bicellar systems have become a useful membrane mimetic since it provides an oriented media for the study of molecules with biological interest, using solid ${ }^{6-9}$ and solution NMR ${ }^{10,11}$. Bicellar systems are formed from mixtures of the dihexanoylphosphatidylcholine (DHPC) and dimiristoylphosphatidylcholine (DMPC) $)^{4,12}$ and occasionally, dipalmitoylphatidylcholine (DPPC $)^{13-15}$. On the other hand, it has been reported that adding a third lipid, with propensity to form a non-lamellar phase, to the DPPC/SM mixture, increases the permeation of cytolitic proteins ${ }^{16}$. Additionally, lipid packing defects seems to be essential for the membrane adsorption of various membrane proteins ${ }^{17,18}$. In this work, we study the effect of low concentration of DHPC over the dynamics of bilayer components in large unilamellar vesicles (LUV) formed with different concentrations of $\mathrm{DPPC} / \mathrm{SM} / \mathrm{DHPC}$, using fluorescence spectroscopy and molecular dynamics (MD) simulations.

\section{EXPERIMENTAL}

All lipids (DPPC, DHPC and SM) were purchased from Avanti Polar Lipids (Alabaster, AL). They were $99 \%$ pure and used as received. Laurdan and 1,6-diphenyl-1,3,5-hexatriene (DPH) were purchased from Sigma-Aldrich and used without further purification. The samples were prepared from DPPC, SM and DHPC stock solutions in chloroform/methanol (4:1). Aliquots of the different solutions were mixed to obtain four systems with the following DPPC/ SM/DHPC proportions: A) $85 / 15 / 0$; B) 80/15/5; C) 75/15/10; D) 70/15/15 mol $\%$. Samples were poured into a glass vial and evaporated to dryness under a nitrogen flow. Multilamellar vesicles (MLV) were obtained by hydration with Tris- $\mathrm{HCl} 20 \mathrm{mM}$ and $140 \mathrm{mM} \mathrm{NaCl}$ buffer solution to give a final lipid concentration of $900 \mu \mathrm{M}$. Large unilamellar vesicles (LUV) were prepared by extruding the MLV suspension. Ten extrusions were performed each time. The extruder was used with polycarbonate filter with $400 \mathrm{~nm}$ pores size (Merck Millipore). Steady-state Laurdan fluorescence emission was acquired with a Perkin-Elmer LS-55 spectrofluorometer. Vesicles ( $900 \mu \mathrm{M}$ lipid) were equilibrated in the presence of $2 \mu \mathrm{M}$ of Laurdan at different temperatures, until the fluorescence emission was stable, typically few hours. Emission spectra were acquired from $365 \mathrm{~nm}$ to $600 \mathrm{~nm}$ with an excitation wavelength of $350 \mathrm{~nm}$.
Laurdan's Generalizated Polarization (GP) was calculated from the following equation ${ }^{19-21}$ :

$$
G P=\frac{I_{440}-I_{490}}{I_{440}+I_{490}}
$$

where $I_{440}$ and $I_{490}$ correspond to fluorescence intensities at those wavelengths.

DPH fluorescence anisotropy was obtained using Perkin-Elmer LS55 spectrofluorometer with polarizer attachment. Samples were prepared as described above. The excitation wavelength was $355 \mathrm{~nm}$ and the emission was observed at $455 \mathrm{~nm}$. Steady-state anisotropy (r) was determined in L-format and was obtained directly from LS software, using the equation ${ }^{22}$ :

$$
r=\frac{I_{V V}-G I_{V H}}{I_{V V}+2 G I_{V H}}
$$

where $I_{V V}$ and $I_{V H}$ are the parallel and perpendicular polarized fluorescence intensities, measured with a vertically polarized excitation light; $I_{H V}$ and $I_{H H}$ are the fluorescence intensities, measured with the excitation light horizontally polarized, and $\mathrm{G}$ is the monochromator correction factor, given by $G=I_{H V} / I_{H H}$

Molecular Dynamics. Four computational boxes were built. Table 1 shows the composition of all systems. The bilayer was solvated with 3611 SPC water molecules ${ }^{23}$. All MD simulations were performed using the program package GROMACS version 4.5.3 ${ }^{24,25}$ in the isothermic-isobaric ensemble. The employed force field was a combination of two potentials: the GROMOS96-53a6 ${ }^{26}$ parameters were used for most interactions of all atoms, i.e. bonds stretching, angles bending, torsions, out of plane bending, and Lennard-Jones interactions. For the Lennard-Jones interactions of the aliphatic chains, the force field developed by Berger et al. was employed 27. The united atom approximation was used for all hydrogen atoms in the aliphatic chains. The Ryckaert-Belleman ${ }^{28}$ potential function was employed to calculate the potential energy of the aliphatic chain torsions. LINCS ${ }^{29}$ was used to constrain the bond lengths of the surfactant chains and SETTLE 30 to restrict the structure of the water molecules. A $1 \mathrm{~nm}$ cut-off was used for the Lennard-Jones potential and the real space electrostatic interactions. Long range electrostatic interactions were calculated using $\mathrm{PME}^{31}$. To maintain the temperature and pressure at constant values of $310 \mathrm{~K}$ (water, salt and surfactant coupled independently) and 1 bar, the weak coupling algorithm of Berendsen was used, with time constants of 0.1 and $1 \mathrm{ps}$, respectively ${ }^{32}$. The time step size in all simulations was $2 \mathrm{fs}$. The neighbor list was updated every 10 time steps. Trajectories of $250 \mathrm{~ns}$ were calculated for all systems. Analyses of trajectories were performed over the last $50 \mathrm{~ns}$ of simulations. Additionally, packing defects at the membrane surface was evaluated calculating the accessible solvent area (ASA) of the lipid headgroups and acyl chains, with the program NACCESS $^{33}$, using a probe radius of $0.14 \mathrm{~nm}$. The ASA was evaluated on the two leaflets of the bilayer. 


\section{RESULTS AND DISCUSSION}

Figure 1a shows the emission spectra of Laurdan dissolved into LUV in system $\mathrm{C}$ at $25^{\circ} \mathrm{C}$ and $47^{\circ} \mathrm{C}$. Laurdan is composed of a naphthalene molecule substituted at the 2-position by dimethylamino and at the 6-position by a lauroyl group. This structure produces a higher dipole moment during the excitation process in the naphthalene moiety. Increasing of dipole moment in the excited state induces a reorientation of dipoles of the solvent. Laurdan's hydrophobic chain allows the solubilization in the bilayer, while the dimethylamine group is located at the interface. Thus, the naphthalene moiety resides at the level of glycerol in contact with the solvent molecules. In the gel phase, the Laurdan's dipolar relaxation is produced in the lipidic environment by a few water molecules, showing an emission maximum centered at $440 \mathrm{~nm}$. In the liquid crystalline state, the larger amount of water molecules present in the Laurdan environment induces a relaxation with an emission maximum centered at 490 $\mathrm{nm}^{34}$.

Fig. 1b displays Laurdan's GP as a function of temperature for the four vesicle systems studied. In figure $1 \mathrm{~b}$ three regions can be observed. First, a region below $40^{\circ} \mathrm{C}$ associated to high $\mathrm{GP}$ values, but in continuous descent with increasing temperature; second, a region between $40^{\circ} \mathrm{C}$ and $43^{\circ} \mathrm{C}$, where a fast decrease of GP is observed; and third, over $43^{\circ} \mathrm{C}$, where a progressive decline of GP is observed. As it has been reported, Laurdan is sensitive to the degree of water penetration into the membrane. GP values above 0.5 and around 0.0 are associated to the gel phase of DPPC and SM, respectively ${ }^{19,35}$. System A presents GP values in the first region around 0.3. Considering that the mixture $\mathrm{DPPC} / \mathrm{SM}$ does not form microdomains ${ }^{36}$, this result indicates that Laurdan is located in a bilayer arrangement, with water penetration somewhere between pure DPPC and pure SM systems. The second region of the same system allows determining the phase transition temperature. For system $\mathrm{A}, \mathrm{Tm}$ is $42^{\circ} \mathrm{C}$. Above $42^{\circ} \mathrm{C}$ the GP decreases to values between 0.0 and -0.1 , typically observed for liquid crystalline state of DPPC and SM phases.

In the presence of DHPC (systems B,C and D), GP values are, in general, lower than for system A, indicating that the penetration of water in these systems occurs in greater extension than in system A. Furthermore, the GP values decrease progressively with the increment in DHPC concentration, suggesting that short-chain lipids would induce a decrease in the bilayer packing due to the higher mobility of DHPC present at the lipid surface. In the first region of system D the GP values are around 0.05 , which are near to the liquid crystalline phase of system A. This indicates that the dynamics of the lipids at the vesicle interface, in both systems, are similar. The temperature range over which the lipid state transition occurs, from gel phase to liquid crystal phase, is broadened. Above the Tm, systems C and D decrease their GP values reaching the same value at $52^{\circ} \mathrm{C}$.
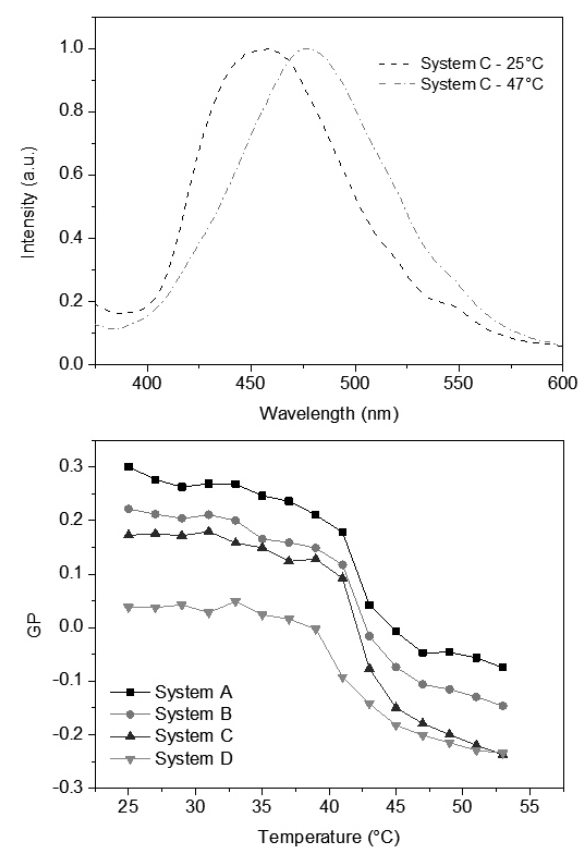

Figure 1. a) Emission spectra of Laurdan solubilized in system $\mathrm{C}$ at $25^{\circ} \mathrm{C}$ and $47^{\circ} \mathrm{C}$. b) GP values for the four systems as a function of temperature.
To sense the effect of DHPC over the hydrophobic region of the lipid bilayer, fluorescence anisotropy of DPH was measured. DPH is a hydrophobic rigid molecule with cylindrical symmetry, solubilized in the acyl chains region of the bilayer. When this probe is excited by polarized light, the dipole emits polarized light in a determined range of angles. Thus, in the gel phase, DPH will have a narrow distribution of angles due to a hindered rotational diffusion. This is observed as high values of fluorescence anisotropy. In the liquid crystalline phase, DPH will have higher rotational diffusion increasing the angles distribution, and therefore, decreasing the fluorescence anisotropy ${ }^{22}$. Figure 2 shows the anisotropy for DPH as a function of temperature for the four systems. It represents a direct measurement of the order of the bilayer. Anisotropy values for system A are very similar to those found for pure DPPC ${ }^{37}$. The increment of DHPC concentration in the lipid mixtures reduces the anisotropy values, indicating that the short-chain lipids diminish the packing of the lipids in the bilayer, increasing the fluidity of the membrane. Interestingly, the systems $\mathrm{C}$ and $\mathrm{D}$ present a liquid crystalline behavior at lower ranges of temperature.

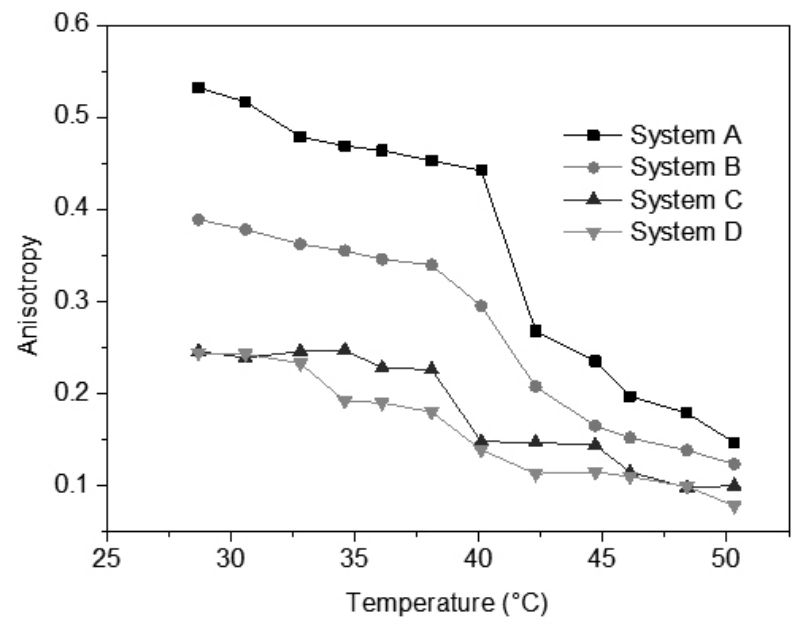

Figure 2. Steady-state fluorescence anisotropy of DPH in the four studied vesicles as a function of temperature.

To obtain detailed information at atomistic level, MD simulations of the four studied systems were performed. Table 1 shows the number of each species present in every simulation box, representing systems A to D. These numbers correspond to the experimental composition.

Table 1. Composition of simulated systems.

\begin{tabular}{|c|c|c|c|c|c|c|}
\hline System & DPPC & SM & DHPC & $\mathrm{H}_{2} \mathrm{O}$ & $\mathrm{Na}+$ & Cl- \\
\hline A & 109 & 19 & 0 & 3611 & 22 & 22 \\
\hline B & 103 & 19 & 6 & 3611 & 22 & 22 \\
\hline C & 96 & 19 & 13 & 3611 & 22 & 22 \\
\hline D & 90 & 19 & 19 & 3611 & 22 & 22 \\
\hline
\end{tabular}

Figure 3 (on the left) shows the top views of the normal to the bilayer surface for systems A to D. The hydrophobic tails are represented in green and the hydrophilic headgroup in blue, respectively. It can be observed that the lipid packing defects are more abundant in the systems with larger concentration of DHPC. To quantify how deep is the penetration of water in the lipid packing defects, we have calculated the accessible solvent area (ASA) for headgroups and acyl chains of the long-chain lipids in all systems (see Table 2). For ordered systems, as the gel phase, ASA values are expected to be lower because the lipid packing occur in larger extension. The increment in the lipid molecular dynamics increases the bilayer fluidity, inducing larger defects in the lipid packing observed as greater values of $\mathrm{ASA}^{38}$. From the simulations, we observe that the ASA values increase with increasing DHPC concentration, suggesting that the extension of the acyl chains exposed to the solvent is produced by a higher fluidity of the bilayer. 

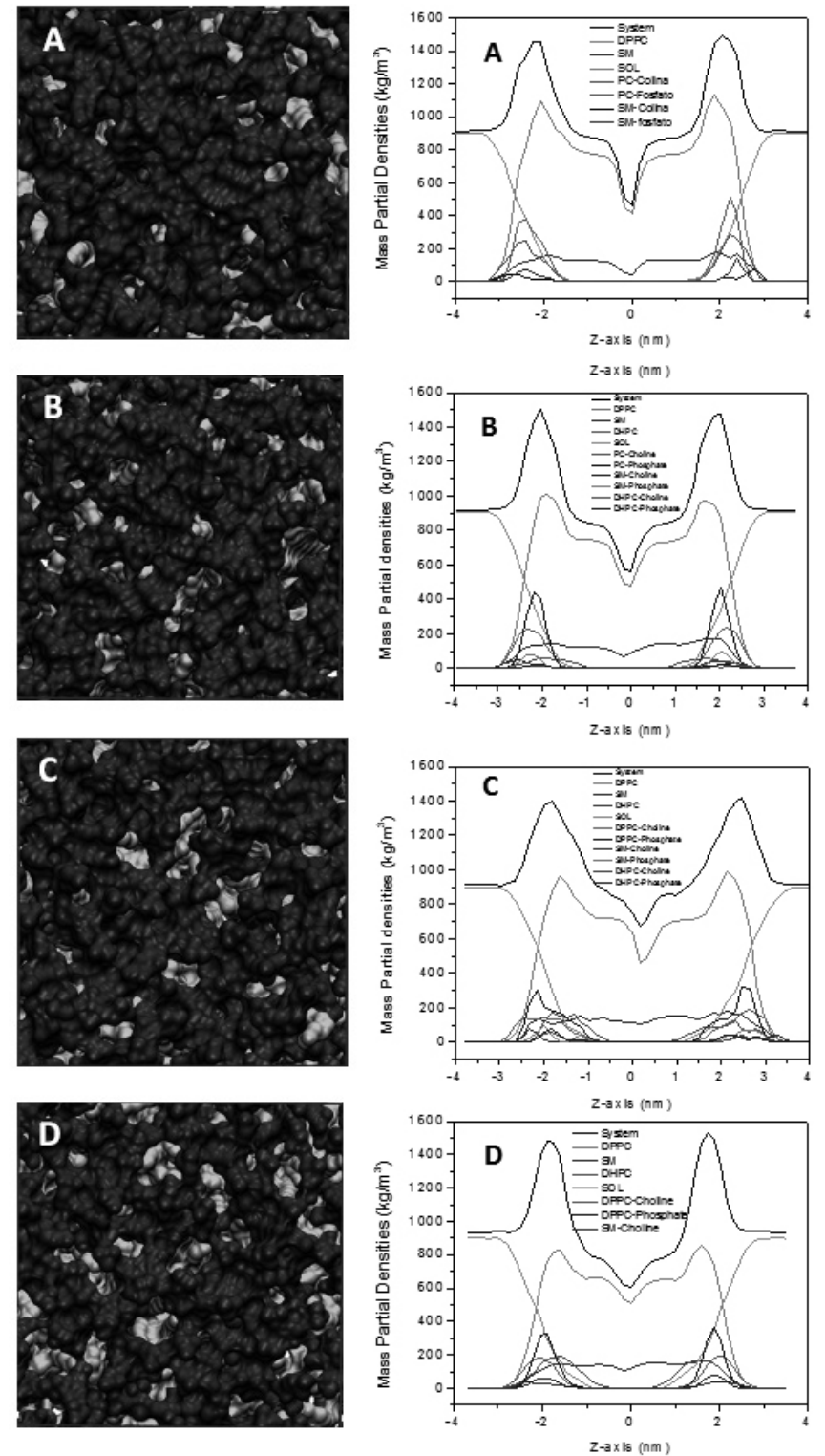

Figure 3. Snapshot of top view of the four systems at 249ns of calculation. Atoms in the headgroups and acyl chains are shown in surface representation in blue and green, respectively.

These results are in agreement with those obtained for DOPC bilayers ${ }^{39}$ and with the experimental results obtained by Laurdan's GP. Additionally, we observe that although DHPC increases the number and size of packing defects in the interfacial region, these defects are not necessarily located along the DHPC molecules, suggesting a global perturbation of the membrane. Similar effect was observed by Vamparys and co-workers, for the mixture of dioleoylphosphocholine (DOPC) and dioleoylglycerol (DOG) ${ }^{39}$.

Figure 3 (on the right) shows the partial densities, along the $\mathrm{Z}$ axis of the box, for DPPC, SM, DHPC, the solvent and the complete system, for the four boxes. Additionally, to evaluate the distance between lipids headgroups in the different leaflets (bilayer thickness) and the thickness of the interface, the solvent, choline and phosphate group mass distributions are presented for the different lipids in all systems. These results are shown in Table 2.
Table 2. Accessible solvent area (ASA) for lipid headgroups and acyl chains, and length of interfacial region and bilayer thickness

\begin{tabular}{|c|c|c|c|c|}
\hline System & $\begin{array}{c}\text { ASA }\left(\mathrm{nm}^{2}\right) \\
\text { Headgroups* }\end{array}$ & $\begin{array}{c}\text { ASA }\left(\mathrm{nm}^{2}\right) \\
\text { Acyl chains }\end{array}$ & $\begin{array}{c}\text { Interface size } \\
(\mathrm{nm})\end{array}$ & $\begin{array}{c}\text { Bilayer } \\
\text { Thickness } \\
(\mathrm{nm})\end{array}$ \\
\hline A & 1.321 & 0.682 & 1.11 & 3.93 \\
\hline B & 1.413 & 0.869 & 1.14 & 3.62 \\
\hline C & 1.425 & 0.884 & 1.29 & 3.6 \\
\hline D & 1.492 & 0.94 & 1.32 & 3.38 \\
\hline
\end{tabular}

*Headgroup includes the phosphatidyl choline of both long-chain lipids, and glycerol group of DPPC and ceramide group of sphingomyelin.

The increment in the interfacial thickness is explained by the observation that packing defects allow water molecules to penetrate deeper inside the bilayer.

The decrease in bilayer thickness could be explained based on the disorder induced by the DHPC lipids. Considering system A, the density profile for DPPC and SM clearly establishes the division between the two leaflets that compose the bilayer. This division progressively starts to disappear with the increase of DHPC concentration. This observation suggests that the increase of disorder in the bilayer facilitates the interdigitation between the two leaflets, reducing the membrane thickness.

Finally, the interior of lipid bilayer was examined by the calculation of order parameters. Figure 4 shows the order parameters for DPPC and SM, in systems A and D, as a function of the carbon position in the chain lipid. System A presents order parameter values typically observed for chain lipids highly ordered like those in gel phases. The calculated values are near to those reported for DPPC in gel state ${ }^{40}$. The origin of the differences in $\mathrm{S}_{\mathrm{CD}}$ values for the first eight carbons could be explained based on the differential

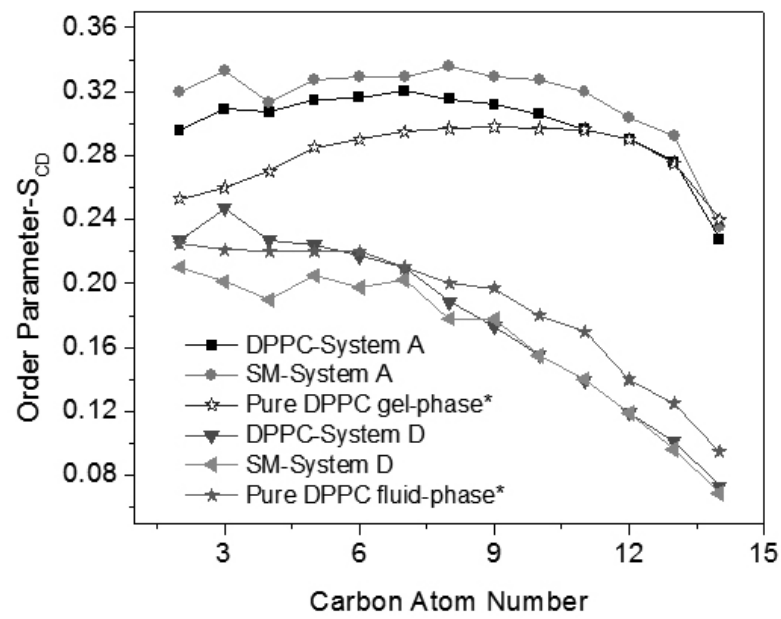

Figure 4. Order parameters of the acyl chains of DPPC and SM. For simplicity, only order parameters for system A and system D are shown. *Values for DPPC, obtained in previous studies, in gel and liquid crystalline states*.

packing between glycerol and ceramide moieties in the DPPC/SM system regarding pure DPPC. On the other hand, the values of the order parameter obtained for system D are lower than those obtained for system A, suggesting that chains in system D are less ordered with values typically observed for liquid crystal state. This behavior is in agreement with previous reports ${ }^{41}$.

\section{CONCLUSIONS}

The inclusion of DHPC in the bilayer induces important disorder on the lipid components of the membrane. Low concentration of DHPC induces packing defects over the interfacial and the hydrophobic region. Furthermore, considering that packing defects are not necessarily located near the DHPC molecules, their incorporation suggests a global perturbation of the membrane 
components. Finally, increasing the concentration of DHPC induces a liquid crystalline behavior in all the systems studied.

\section{ACKNOWLEDGEMENTS}

The authors are pleased to acknowledge financial support from Fondecyt grant No. 11085049 and CYTED-212RT0467.

\section{REFERENCES}

1.- K. Simons,E. Ikonen, Nature 387, 569, (1997)

2.- K. Simons,J.L. Sampaio, Cold Spring Harb. Perspect Biol. 3, (2011)

3.- D.A. Brown,E. London, J.Membr.Biol. 164, 103, (1998)

4.- P. Ram,J.H. Prestegard, Biochim.Biophys.Acta 940, 289, (1988)

5.- H.T. McMahon,J.L. Gallop, Nature 438, 590, (2005)

6.- C.R. Sanders,J.P. Schwonek, Biochemistry 31, 8898, (1992)

7.- C.R. Sanders, G.C. Landis, Biochemistry 34, 4030, (1995)

8.- A.A. Nevzorov,S.J. Opella, J. Magn. Resn. 185, 59, (2007)

9.- T. Cui, C.G. Canlas, Y. Xu, P. Tang, Biochim.Biophys.Acta 1798, 161, (2010)

10.- G. Wang, Curr.Protein Peptide Sci. 9, 50, (2008)

11.- H.J. Kim, S.C. Howell, W.D. Van Horn, Y.H. Jeon, C.R. Sanders, Prog Nucl Magn Reson Spectrosc 55, 335, (2009)

12.- L. Barbosa-Barros, A. De La Maza, P. Walther, A.M. Linares, M. Feliz, J. Estelrich, O. Lopez, J. Microsc (Oxf) 233, 35, (2009)

13.- G. Rodriguez, L. Rubio, M. Cocera, J. Estelrich, R. Pons, A. De La Mazaa, O. Lopez, J.Pharm.Pharmacol. 62, 791, (2010)

14.- L. Rubio, G. Rodriguez, C. Alonso, C. Lopez-Iglesias, M. Cocera, L. Coderch, A. De la Maza, J.L. Parra, O. Lopez, Soft Matter 7, 8488, (2011)

15.- T.L. Lin, C.C. Liu, M.F. Roberts, S.H. Chen, J.Phys.Chem. 95, 6020, (1991)

16.- C.A. Valcarcel, M. Dalla Serra, C. Potrich, I. Bernhart, M. Tejuca, D. Martinez, F. Pazos, M.E. Lanio, G. Menestrina, Biophys.J. 80, 2761, (2001)

17.- R.M. Epand, Biochim.Biophys.Acta 1376, 353, (1998)

18.- B. Antonny, Annu. Rev. Biochem 80, 101, (2011)

19.- T. Parasassi, G. De Stasio, G. Ravagnan, R.M. Rusch, E. Gratton,
Biophys.J. 60, 179, (1991)

20.- M. Cubillos, E. Lissi, E. Abuin, J. Chil. Chem. Soc. 51, 825, (2006)

21.- E. Berrios, A.L. Zanocco, E. Lemp, G. Guenther, J. Chil. Chem. Soc. 53, $1728,(2008)$

22.- J. R. Lakowicz. , Principles of Fluorescence Spectroscopy, Springer, New York, 2007.

23.- H. J. C. Berendsen, J. P. M. Postma, W. F. van Gunsteren and J. Hermans. , Intermolecular Forces, D.Reidel Publishing Company, 1981.

24.- W.F. Vangunsteren,H.J.C. Berendsen, Angew. Chem. Int. Ed 29, 992 , (1990)

25.- H.J.C. Berendsen, D. Vanderspoel, R. Vandrunen, Comput.Phys.Commun. 91, 43, (1995)

26.- C. Oostenbrink, A. Villa, A.E. Mark, W.F. Van Gunsteren, J. Comp Chem. 25, 1656, (2004)

27.- O. Berger, O. Edholm, F. Jahnig, Biophys.J. 72, 2002, (1997)

28.- J.P. Ryckaert,A. Bellemans, Faraday Discuss. Chem. Soc. 66, 95, (1978)

29.- B. Hess, H. Bekker, H.J.C. Berendsen, J.G.E.M. Fraaije, J. Comp. Chem. 18, 1463, (1997)

30.- S. Miyamoto,P.A. Kollman, J. Comput. Chem. 13, 952, (1992)

31.- T. Darden, D. York, L. Pedersen, J.Chem.Phys. 98, 10089, (1993)

32.- H.J.C. Berendsen, J.P.M. Postma, W.F. van Gunsteren, A. DiNola, J.R.J. Haak, Chem. Phys. 81, 3684, (1984)

33.- S.J. Hubbard,J.M. Thornton, NACCESS. Department of Biochemistry and Molecular Biology, University College London, London,UK, (1993)

34.- S.A. Sanchez, M.A. Tricerri, G. Gunther, E. Gratton, Modern Research and Educational Topics in Microscopy 1, 1007, (2007)

35.- L.A. Bagatolli, T. Parasassi, G.D. Fidelio, E. Gratton, Photochem. Photobiol. 70, 557, (1999)

36.- P. Niemela, M.T. Hyvonen, I. Vattulainen, Biophys.J. 87, 2976, (2004)

37.- I. Konopasek, J. Vecer, K. Strzsalka, E. Amler, Chem.Phys.Lipids 130, 135, (2004)

38.- H. Cui, E. Lyman, G.A. Voth, Biophys.J. 100, 1271, (2011)

39.- L. Vamparys, R. Gautier, S. Vanni, W.F.D. Bennett, D.P. Tieleman, B. Antonny, C. Etchebest, P.F.J. Fuchs, Biophys.J. 104, 585, (2013)

40.- S. Leekumjorn,A.K. Sum, Biochim.Biophys.Acta 1768, 354, (2007)

41.- T. Mehnert, K. Jacob, R. Bittman, K. Beyer, Biophys.J. 90, 939, (2006) 